\title{
NOTAS SOBRE MACROMICETES DE LA ZONA CENTRAL DE CHILE
}

\section{(Notes on macromycetes from areas of central Chile)}

\author{
Hugo Madrid \\ Tecnólogo Médico. Fono: 6729902, \\ e-mail: hugo.madrid @gmail.com
}

Palabras claves: Basidiomycota, Chile central Key Words: Basidiomycota, central Chile.

\section{RESUMEN}

Se realizó un estudio taxonómico de carpóforos de macromicetes recolectados entre los meses de junio y noviembre de 2005 en áreas urbanas y semi rurales de la zona central de Chile. Este trabajo es un reporte preliminar de las especies determinadas (entre ellas Schizophyllum commune) con notas sobre algunos aspectos ecológicos y de interés médico humano.

\section{INTRODUCCION}

En Chile las particulares características geográficas y climáticas determinan la existencia de una interesante micota, sobre la cual existe limitado conocimiento y cuyo estudio merece especial interés, tanto a nivel básico como aplicado, debido a la presencia de especies de gran relevancia en los ámbitos de la patología humana, forestal y agrícola (Mujica et al., 1980; Piontelli, 1995). En estudios previos realizados por Singer (1969), Lazo (2001), Garrido (1988) y Valenzuela (2003), se han señalado aspectos taxonómicos, ecológicos y el empleo de diversos macromicetes que fructifican en distintos ambientes ecológicos de Chile.

El autor de la presente nota, con el objetivo de aportar nuevos datos ecológico y de distribución de hongos superiores chilenos, de importancia en salud pública, ha recolectado diversos basidiocarpos (entre los meses de junio y noviembre de 2005), en sectores urbanos y semirurales de la zona central de Chile, incluyendo la Región Metropolitana (ciudades de Santiago y Buin) y la localidad de Guacarhue de la Sexta Región del país.

\section{MATERIAL Y METODOS}

Alguno de los basidiocarpos fueron recolectados en la ciudad de Santiago, capital de Chile, localizada en la zona central del país. La ciudad de Santiago es un área altamente urbanizada e industrializada, posee un clima

\begin{abstract}
A carries out a taxonomic study of carpophore recollected in urban and rural areas of Central Chile, between June and November of 2005. This work is a preliminary report of those determinates species (between these Schizophyllum commune)with notes on some ecological and of human medical interest aspects.
\end{abstract}

templado cálido con lluvias invernales y una estación seca prolongada, con un promedio de precipitación anual de 312.5 mm (Dirección Meteorológica de Chile, 2005). En esta ciudad, algunos lugares favorables para recolectar carpóforos son Plaza Italia, los parques: De Los Reyes, Quinta Normal y Forestal, todos ellos caracterizados por la presencia de amplios prados y predominio de especies arbóreas alóctonas. Por su parte, las localidades de Buin y Guacarhue son zonas semi rurales de características climáticas similares a las descritas para Santiago, en las cuales existe un importante desarrollo del rubro agrícola, con extensos terrenos dedicados al cultivo de hortalizas y frutales.

El estudio taxonómico de los carpóforos se llevó a cabo de acuerdo a lo indicado por Moreno et al. (1986) y Lazo (2001), las características macro y microscópicas determinadas (color, olor, sabor, forma de píleo y estípite, esporas, basidios, cistidios, hifas, etc.), fueron comparadas con descripciones de las especies que aparecen en los trabajos antes citados y en otros textos especializados en taxonomía de macromicetes, entre otros Singer (1960), Smith (1963), Pulido (1983), Johnston \& Buchanan (1995) y Valenzuela (1998). El material estudiado se conserva en el herbario particular del autor.

\section{RESULTADOS Y DISCUSION}

En los lugares de Santiago (Plaza Italia, los parques: De Los Reyes, Quinta Normal y Forestal), al 
igual que en calles y jardines de esta ciudad, los macromicetes más frecuentes son especies del género Coprinus (Fig. 1), especialmente C. micaceus (Bull.: Fr.) Fr., el cual fructifica en suelos, generalmente cerca de madera en descomposición o de las raíces de algunos árboles. En Plaza Italia y Parque Forestal, fue posible recolectar basidiomas de esta especie desde junio hasta inicios de noviembre, fructificando con mayor abundancia en el período más lluvioso. Otras especies, recolectadas ocasionalmente, fueron C. disseminatus (Pers. ex Fr.) Gray, en suelos de calle San Pablo y Psathyrella candolleana Maire in Maire et Werner, en jardines del Instituto de Salud Pública. Por su parte en prados del Parque De Los Reyes, ubicado en el sector céntrico de Santiago, las frágiles y efímeras fructificaciones de Bolbitius vitellinus (Pers. ex Fr.) Fr. (Fig. 2), destacaron por su frecuencia apareciendo en abundancia a las pocas horas del inicio de las precipitaciones. En este mismo lugar fueron recolectados carpóforos de Agaricus sp. y Coprinus comatus (Müller. Fr.) S. F. Gray. Otro lugar de Santiago particularmente interesante para la recolección de setas es el cerro San Cristóbal, el cual posee una mezcla de flora nativa y alóctona, con suelos ricos en materia vegetal en descomposición, permitiendo el desarrollo de numerosos macromicetes. En este lugar se recolectaron, entre los meses de septiembre y octubre, Cyathus olla Batsch ex Pers., Geastrum fornicatum (Huds.) Fr. y hongos pertenecientes a los géneros Morchella (Ascomycota), Mycena, y Ramaria.

En las localidades de Buin y Guacarhue se encontraron numerosos Agaricales, incluyendo Coprinus spp. y algunas setas comestibles. En Buin, en suelos de una plantación de nogales fueron recolectados Volvariella speciosa (Fr. ex Fr.) Singer y en troncos de algunos árboles A. aegerita, ambas especies son muy cotizadas por la gastronomía local. Otro basidiomycete común en esta zona es Lepista nuda (Bull. ex Fr.) Cooke (Fig. 3 y 4), la cual es conocida con el nombre común de «callampa morada» (Lazo, 1982) o «callampa violeta» (Espinosa, 1929), generalmente es considerada comestible, siendo muy apreciada en algunos países, pero que, sin embargo, se ha asociado ocasionalmente a trastornos digestivos (Moreno, 1986). Aparentemente, la literatura nacional no registra casos de intoxicaciones debidas a la ingesta de fructificaciones de $\mathbf{L}$. nuda. Sin embargo, en archivos de la Sección de Botánica del Museo de Historia Natural de Chile, el autor tuvo la oportunidad de encontrar una interesante nota , que dataría de mediados del siglo pasado, y que fue escrita por el célebre micólogo Marcial Espinosa, quien señala, textualmente, al referirse a esta especie, mencionada como Tricholoma personatum (Fr). Quél.: «En Chile venenoso, según las observaciones del Prof. Dr.
Ramón Valdivieso D. en su clínica del Hospital San Vicente en junio de 1948... La mayoría de los micólogos de otros países lo consideran comestible...». Es posible que exista conocimiento entre los habitantes de Buin sobre la eventual toxicidad de $\boldsymbol{L}$. nuda, dado que al consultar a trabajadores agrícolas de esta localidad sobre cómo seleccionan los hongos silvestres que consumen, uno de los principales criterios señalados por ellos fue rechazar la «callampa morada», la cual consideran venenosa.

Por otra parte, debido al desarrollo de una importante actividad ganadera dedicada a la crianza de equinos y bovinos, además del enriquecimiento de los suelos con heces de estos animales, es posible observar en sectores de Chile central, una variedad de Basidiomycetes coprófilos, pertenecientes a los géneros Psilocybe y Panaeolus, incluyendo numerosas especies tóxicas (Lazo, 2001). En Guacarhue a mediados del mes de septiembre fue recolectado Panaeolus sphinctrinus (Fr.) Quélet, el cual fructificaba en estiércol bovino. De acuerdo a Moreno et al. (1986), en los basidiocarpos de este hongo se ha detectado cantidades importantes de psilocina y psilocibina, compuestos que al interactuar con receptores de neurotransmisores, pueden originar intoxicaciones de diversa gravedad, cuyas manifestaciones clínicas van desde delirios y alucinaciones hasta la muerte. En Buin, a fines del mes de agosto, fueron recolectados desde suelo contaminado con estiércol Psilocybe sp., y Panaeolus retirugis (Fr.) Quélet otra especie considerada tóxica (Valenzuela, 1996; Lazo, 2001).

En Santiago, Buin y Guacarhue desde madera, de la corteza de álamos, espinos y otros árboles vivos o muertos fueron recolectadas en forma frecuente basidiocarpos de Schizophyllum commune Fr.: Fr. (Fig. 5), esta especie tiene una distribución cosmopolita, sus fructificaciones en los sustratos antes citados son fácilmente reconocibles por sus carpóforos en forma de concha o abanico, sésiles, de color grisáceos, sus láminas son gruesas, bifurcadas desde los bordes y de color rosado- grisáceo. La literatura reconoce a $\boldsymbol{S}$. commune como un agente emergente de enfermedades oportunistas en el hombre, implicado en casos de sinusitis (Ciferri et al., 1956; Kern \& Uecker, 1986; Catalano et al., 1990; Rosenthal et al., 1992), micosis broncopulmonar alérgica (Kamei et al., 1994), meningitis (Batista et al., 1955), lesiones ulcerosas del paladar (Restrepo et al., 1973), bola fúngica pulmonar (Sigler et al., 1995), absceso cerebral (Rihs et al., 1996) y otras patologías (Kligman, 1950; Amitami, et al., 1996). Además, se ha reportado un caso de infección granulomatosa subcutánea en un perro (Kano et al., 2002). En los tejidos de mamíferos, la morfología de S. commune es variable, presentándose como un micelio hialino, septado, ramificado donde los elementos de valor 

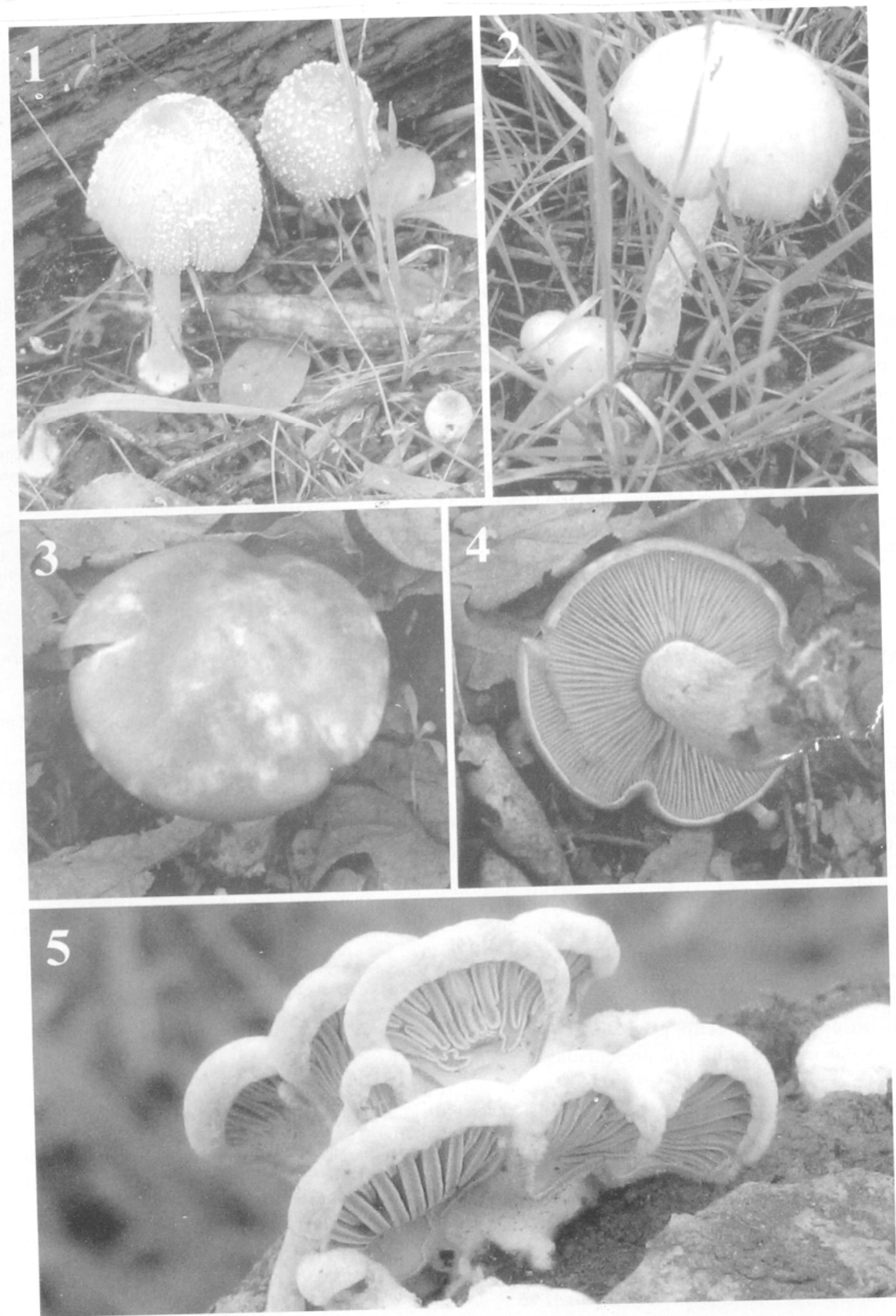

Figura 1.- 1. Coprinus sp. (píleos de 21 a $30 \mathrm{~mm}$ de diámetro). 2. Bolbitius vitellinus (píleos de 16-50 mmm de diámetro). 3-4. Lepista nuda (píleo de $45 \mathrm{~mm}$ de diámetro). 5. Schizophyllum commune (basidiomas de 5-20 x 7-12 mm). 
diagnóstico como fíbulas y espículas pueden hallarse presentes o no, por lo cual, al examen microscópico directo de muestras clínicas, esta especie puede presentar gran similitud con otros hongos oportunistas como Aspergillus y Fusarium (Sigler et al., 1995). Por otra parte, se ha descrito que algunos aislamientos de $\boldsymbol{S}$. commune producen basidiocarpos típicos in vitro muy tardíamente, o no llegan a producirlos, pudiendo originar estructuras tubulares estériles («formas medusoides»), o simplemente permaneciendo bajo la forma de un micelio blanco, algodonoso, sin fructificaciones, por lo cual se ha requerido el empleo de metodologías especiales, como técnicas de cruzamiento (Sigler et al, 1999) o de biología molecular para una identificación definitiva de esta especie (Sigler et al., 1995; Buzina et al., 2001).

En Chile, aunque no se han reportado casos de infección humana por $\boldsymbol{S}$. commune, en consideración a su presencia en el ambiente y a la creciente población de pacientes inmunosuprimidos, este hongo debería tomarse en cuenta como un potencial agente de micosis oportunistas en nuestro medio.

\section{AGRADECIMIENTOS}

Al Profesor Waldo Lazo de la Facultad de Ciencias, Universidad de Chile, por sus valiosos comentarios y colaboración en la determinación del material estudiado. A la Sra. Elizabeth Barrera e Inés Meza de la Sección Botánica del Museo de Historia Natural de Chile por facilitar bibliografía especializada y por la generosa acogida en su laboratorio, donde se realizó gran parte de este trabajo. Al T.M. Sr. Cesar Pino, por su participación en la recolección de carpóforos y su constante colaboración en numerosos aspectos técnicos. Al Dr. Victor Silva de la Facultad de Medicina, Universidad de Chile, por fructíferos dos años de aprendizaje y trabajo científico en su laboratorio, al Dr. Eduardo Valenzuela por la revisión y comentarios en el manuscrito y al Señor Francisco Rojas y Jaime López Rousseau, quienes han hecho posible el registro fotográfico de las especies fúngicas presentadas.

\section{REFERENCIAS}

Amitami, R.; Nishimura, K.; Niimi, A.; Kobayashi, H.; Nawada, R.; Murayama, T.; Taguchi, H.; Kuze, F. (1996). Bronchial mucoid impaction due to the monokaryotic mycelium of Schizophyllum commune. Clin. Infect. Dis. 22:146-148

Batista, A.; Maia, H. da S. \& Alecrim, I. Da C. (1955). Basidioneuromycosis on man. An. Soc. Biol. Pernambuco 13:52-60

Buzina, W.; Lang-Loidolt, D.; Braun, H.; Freudenschuss, K.; Stammberger, H. (2001). Development of molecular methods for identification of Schizophyllum commune from clinical samples. J. Clin. Microbiol. 39:2391-2396
Catalano, P.; Lawson, W.; Bottone, E. \& Lebenger, J. (1990). Basidiomycetous (mushroom) infection of the maxillary sinus. Otolahyngol. Head Neck Surg. 102:183-185

Ciferri, R.; Batista, A. \& Campos, S. (1956). Isolation of Schizophyllum commune from sputum. Atti Ist. Bot. Lab. Crittogam. Univ. Pavia 14:3-5

Dirección Meteorológica de Chile (2005). www.meteochile.cl

Espinosa, M.. (1929). Contribución al conocimiento de los hongos chilenos. Bol. Mus. Nac. Chile. 12:127-138

Garrido, N. (1988). Agaricales s.l und ihre in den Nothofagus Wäldern mittelciles. J. Cramer, Berlin - Stuttgart.

Johnston , P. \& Buchanan P. (1995). The genus Psilocybe (Agaricales) in New Zealand. N. Z. J.Bot. 33:379-388

Kamei, K.; Unno, H.; Nagao, K.; Kuriyama, T.; Nishimura, K.; Miyaji, M. (1994). Allergic bronchopulmonary mycosis caused by the basidiomycetous fungus Schizophyllum commune. Clin. Infect. Dis. 18:305-309

Kano, R.; Oomae, S.; Nakano, Y.; Minami, T.; Sukikara, M.; Hasegawa A.; Nakayama, T. (2002). First report of Schizophyllum commune from a dog. J. Clin. Microbiol. 40(9): 3535-3537.

Kern, M.E. \& Uecker, F.A. (1986). Maxillary sinus infection caused by the homobasidiomycetous fungus Schizophyllum commune. J. Clin. Microbiol. 23:1001-1005

Kligman, A. (1950). A basidiomycete probably causing onychomycosis. J. Investig. Dermatol. 14:67-70

Lazo, W. (1982). Introducción al estudio de los hongos superiores. Boletín Micológico 1:19-30

(2001). Hongos de Chile. Atlas Micológico. Facultad de Ciencias Universidad de Chile. Santiago.

Moreno, G., Manjón, J. L., \& Zugaza, A. (1986). La guía incafo de los hongos de la Península Ibérica. Vol. I y II. Incafo Madrid. España.

Mujica, F., Vergara, C., \& Oehrens, E. (1980). Flora fungosa chilena. Segunda edición. Santiago, 308 p.

Piontelli, E. (1995). Historia de la micología chilena. Rev. Iberoam. Micol. 12:86-89

Pulido, M. (1983). Estudios en Agaricales colombianos. Instituto de Ciencias Naturales, Universidad Nacional, Colombia.

Restrepo, A.; Greer, A.; Robledo M.; Osorio, O.; Mondragon, H. (1973). Ulceration of the palate caused by a basidiomycete, Schizophyllum commune. Sabouraudia 11:201-204

Rihs, J.; Padhye, A. \& Good C. (1996). Brain abscess caused by Schizophyllum commune: an emerging basidiomycete pathogen. J. Clin. Microbiol. 34:1628-1632

Rosenthal, J.; Katz, R.; Dubois D.; Morrisey, A.; Machicao, A. (1992). Chronic maxillary sinusitis associated with the mushroom Schizophyllum commune in a patient with AIDS. Clin. Infect. Dis. 14:46-48 
Sigler, L.; De la Maza, L.; Tan, G.; Egger, K.; Sherburne R. (1995). Diagnostic difficulties caused by a nonclamped Schizophyllum commune isolate. J. Clin. Microbiol. 33:1979-1983

Sigler, L.; Bartley J.; Parr D. \& Morris A. (1999). Maxillary sinusitis caused by a medusoid form of Schizophyllum commune. J. Clin. Microbiol. 37:3395-3398

Singer, R. (1960). Sobre algunas especies de hongos presumiblemente psicotrópicos. Lilloa. 30:119-127

Singer, R. (1969). Mycoflora Australis. Lehre Verlag von J. Cramer.
Smith, A. (1963). The mushroom hunter's field guide revised and enlarged. Ann Arbor The University of Michigan Press. U.S.A.

Valenzuela, E. (1996). Agaricales sensu lato tóxicos recolectados en la X región de Chile. Boletín Micológico 11:1-10

(1998). Guía de campo para setas (Agaricales) de la Isla Teja, Valdivia.

(2003). Hongos comestibles silvestres colectados en la X Región de Chile. Boletín Micológico 18:1-14 